\title{
Regulation of Anti-Inflammatory Gene Expression in Vascular Endothelial Cells by EPAC1
}

\author{
Timothy M Palmer ${ }^{1}$ and Stephen J Yarwood ${ }^{2 *}$ \\ ${ }^{1}$ Bradford School of Pharmacy, University of Bradford, West Yorkshire, BD7 1DP, United Kingdom
}

${ }^{2}$ Institute of Molecular, Cell and Systems Biology, College of Medical, Veterinary and Life Sciences, University of Glasgow, Glasgow G12 8QQ, United Kingdom

"Corresponding author: Stephen J Yarwood, Institute of Molecular, Cell and Systems Biology, College of Medical, Veterinary and Life Sciences, University of Glasgow, Glasgow G12 8QQ, United Kingdom, Tel: +441413303908; Fax: +441413305481; E-mail: Stephen.Yarwood@glasgow.ac.uk

Received date: Sep 16, 2015; Accepted date: Sep 18, 2015; Published date: Sep 23, 2015

Copyright: @2015 Palmer TM. This is an open-access article distributed under the terms of the Creative Commons Attribution License, which permits unrestricted use, distribution, and reproduction in any medium, provided the original author and source are credited.

\begin{abstract}
Suppressor of cytokine signalling 3 (SOCS3) is a potent inhibitor of pro-inflammatory pathways involved in atherogenesis and the development of neo-intimal hyperplasia $(\mathrm{NIH})$, which contributes to the in-stent re-stenosis responsible for the failure of percutaneous coronary intervention (PCI) procedures. We have shown that cyclic AMP sensor EPAC1 triggers induction of the SOCS3 gene in vascular endothelial cells (VECs), thereby attenuating interleukin 6 (IL-6)-mediated pro-inflammatory signalling. We propose that EPAC1 localisation to the nuclear pore controls cyclic AMP-mediated activation of a C/EBP $\beta / C-J u n$ transcriptional complex, leading to SOCS3 induction and suppression of pro-inflammatory signalling. Future work in this area will involve an integrated approach to determine the wider significance of the EPAC1-C/EBPß/C-Jun pathway in controlling human VEC function and identify new therapeutic targets for management of chronic inflammation in vascular settings.
\end{abstract}

\section{Background}

Atherosclerosis is a serious CVD, which arises from chronic localised inflammation at coronary and carotid arterial branch points [1], and remains the principle cause of death in the developed world despite changes in lifestyle and the wide-spread use of antihypertensive and lipid- lowering drugs (http://www.who.int/). Atherogenesis involves a diet-induced propagation of proinflammatory responses leading to the formation of plaques characterised by cholesterol deposition, fibrosis, remodelling and switching of VECs from an anti-coagulant/anti-inflammatory to a prothrombotic/pro-inflammatory phenotype. If untreated, these lesions either occlude vessels or trigger their rupture, resulting in the formation of thrombi that cause myocardial infarction or stroke. Surgical treatment for atherosclerosis typically involves angioplasty, where arterial plaques are removed and a stent is introduced to maintain blood flow. However, in approximately $25-50 \%$ of cases, mechanical injury during angioplasty can lead to $\mathrm{NIH}$, characterised by localised inflammation and proliferation of vascular smooth muscle cells (VSMCs), thereby precipitating stent failure and myocardial infarction [2]. The increased inflammatory activity associated with atherosclerosis and NIH is partially brought about by increased levels of pro-inflammatory cytokines in the circulation, particularly IL-6 $[3,4]$. Sustained IL-6 production is involved in chronic, low-level vascular inflammation that leads to neointimal thickening [5], vascular dysfunction [6], hypertension [7] and increased risk of myocardial infarction [3]. Indeed, IL- 6 has been detected in atherosclerotic plaques [8] and increases in IL6 in elderly patients are associated with a two-fold increase in both cardiovascular and other causes of mortality [9]. IL-6 affects vascular endothelial cells (VECs) by triggering counter-productive angiogenesis, through vascular endothelial growth factor (VEGF) production [10], and increasing the secretion of chemokine's, like monocyte chemo-attractive protein 1 (MCP-1) [11], that recruit monocytes to the inflamed endothelium.
Given this, there is now a clear need to understand the mechanisms regulating the control of pro-inflammatory IL-6 signalling in VECs if we are to devise new and effective strategies to combat atherosclerosis and $\mathrm{NIH}$.

Signalling by IL- 6 occurs through the IL- 6 receptor complex, composed of an IL-6-binding a chain (IL-6Ra) and gp130, which interacts with IL-6Ra [12]. IL-6 has been reported to exert both inflammatory and anti-inflammatory actions [13] and a single nucleotide polymorphism (SNP; Asp358Ala) has been identified in the IL-6R, which reduces inflammation and the risk of developing coronary heart disease (CHD) [14,15], although the mechanisms for this remain to be determined [16]. It is IL-6 receptor "trans- signalling" [17] that is thought to underlie the pro-inflammatory actions of IL-6 in a variety of diseases, including atherosclerosis [18]. During transsignalling, IL-6 binds to soluble forms of IL-6R (sIL-6R) allowing activation of gp130, even in cells that do not normally express IL-6Ra, such as VECs [17]. Since gp130 is present on all cells, trans-signalling therefore dramatically increases the range of cell targets for IL-6 signalling to include VECs [17]. Consequently, binding of the IL-6/ sIL-6R complex to gp130 on VECs, leads to receptor clustering and activation of the JAK-STAT3 and ERK, MAPK and PI3K signalling pathways. Of these, it is activated STAT3 that then homodimerises and translocates to the nucleus, where it acts as a transcription factor for the induction of pro-inflammatory IL-6-responsive genes, such as MCP-1 and VEGF [11,19].

\section{Inhibition of Vascular Inflammation by SOCS3}

Clearly, regulation of pro-inflammatory IL-6 signalling is vital to prevent chronic inflammation. Understanding the mechanisms controlling IL-6 signalling may therefore pave the way to the development of new strategies to combat vascular inflammation. In this light, one important mechanism for down-regulating JAK-STAT3 signalling is via the suppressor of cytokine signalling (SOCS) family of 
proteins [20], which are often induced directly by the same JAK-STAT pathway they inhibit, forming a classical negative feedback loop [21]. For example, SOCS3 binds to JAK-phosphorylated receptors, via the SOCS3 SH2 domain, thereby inhibiting JAK activity and, consequently, activation of STATs 1 and 3 [22]. SOCS3 then also targets multiple $\mathrm{SH} 2$-bound proteins for proteasomal degradation [22], with proteolytic targets including JAK2 [23]. Consistent with its role as a negative regulator of inflammatory signalling, SOCS3 expression is localised to atherosclerotic plaques [24,25] and SOCS3 knockdown in apoE-/mice increases STAT activation and inflammatory gene expression in aorta, leading to enhanced atherogenesis [25]. Moreover, IL-6 has been reported to promote acute and chronic inflammatory disease in the absence of SOCS3 [26] and conditional deletion of the SOCS3 gene in vascular endothelial cells results in pathological angiogenesis [27]. In contrast, overexpression of SOCS3, or introduction of SOCS-derived peptides in to cells, has been shown to suppress JAK/STAT3 signalling, acute inflammation and the development of atherosclerosis and $\mathrm{NIH}$ effectively illustrating the important protective role of SOCS3 [28-30].

Clearly, novel treatments based on the regulation of SOCS3 levels in cells could have value in the treatment of diseases like atherosclerosis, where there is hyper-activation of JAK-STAT3 signalling. In this respect, the ubiquitous second messenger, cyclic AMP, which is synthesised in VECs in response to Gsa-coupled G-protein-coupled receptor (GPCR) activation, plays a key role in controlling SOCS3 induction and limiting cytokine action; indeed, we have defined a role for the cyclic AMP-activated guanine nucleotide exchange factor (GEF), exchange protein activated by cyclic AMP (EPAC1) [31,32], as a key mediator of SOCS3 induction [31] and a central controller of antiinflammatory processes in VECs $[33,34]$. EPAC1 mediates at least three anti-inflammatory signalling pathways in VECs; namely, downregulation of IL-6- and STAT3-mediated inflammatory processes [31], which occurs through C/EBP transcription factor-dependent induction of the SOCS3 gene [32], limiting vascular permeability through EPAC1-mediated activation of integrins, involved in cell spreading and adhesion of VECs to the basement membrane [35], and promotion of endothelial barrier function through actin [36-40] and microtubule-dependent [41] cell-cell junction formation through stabilisation of VE-cadherin-mediated adhesion [42]. Overall, the involvement of EPAC1 in multiple anti-inflammatory processes in VECs presents an effective model in which to study how distinct cellular processes may interact to present a co-ordinated program of "protection" against inflammatory stimuli.

\section{Hypothesis}

We must now determine the cellular actions of EPAC1 that are linked to SOCS3 induction and inhibition of IL-6 signalling. We propose that EPAC1 may serve as a potential target for future antiinflammatory drug development, with a reduced risk of side effects, based on the following observations

- EPAC1 promotes SOCS3 induction independently of potentially harmful JNK and ERK 1 and 2, MAP kinase signalling pathways, which are normally triggered by changes in global cyclic AMP (Figure 1) [43,44].

- SOCS3 induction by EPAC1 requires C/EBP $\beta$ and c-Jun transcription factors [32,44], which directly interact with the SOCS3 promoter and may therefore serve as a point of integration for EPAC1-regulated signalling to enable effective SOCS3 gene induction in VECs (Figure 2) [44].
- A key AP1 transcription factor binding site is required for EPAC1 to activate the SOCS3 promoter [44].

- C-Jun is constitutively associated with the AP1 site [44], whereas $\mathrm{C} / \mathrm{EBP} \beta$ is recruited to the SOCS3 promoter following EPAC1 activation [32].

- EPAC1 is mainly associated with the nuclear compartment of fractionated HUVECs where it co- localises with the SUMO1 E3 ligase, RanBP2 (results not shown), which was previously identified as an EPAC1 binding protein [45,46].

- The nuclear targeting domain within EPAC1 as being within amino acids $764-838$ in the GEF domain [47]. This domain contains two areas that are unique to EPAC1 and not found in EPAC2. Since EPAC2 is not nuclear targeted to the same degree as EPAC1 [45] then we predict that these areas contain the key determinants for nuclear targeting of EPAC1.

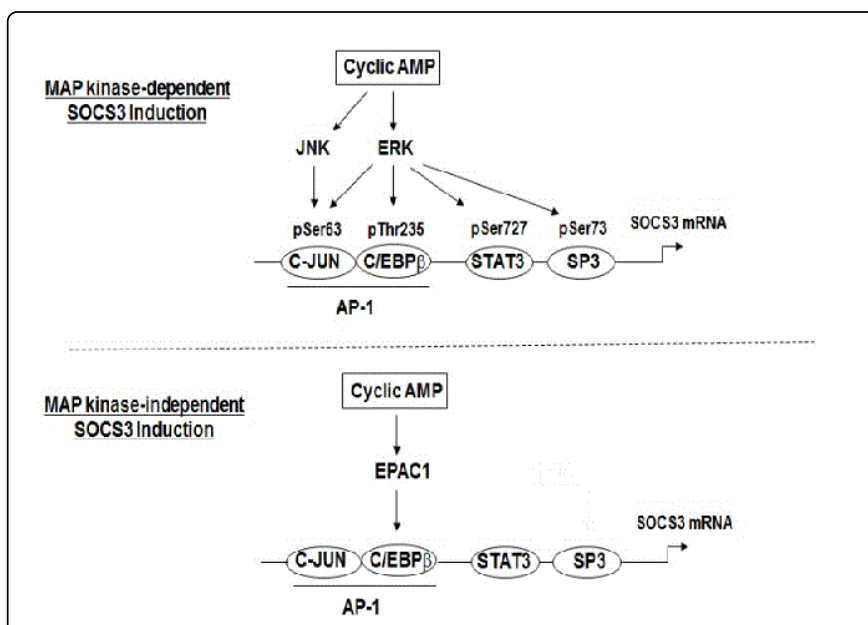

Figure 1: MAP kinase-dependent and -independent control of SOCS3 Gene Induction by cyclic AMP Maximal induction of the SOCS3 gene by cyclic AMP in VECs requires input both EPAC1(lower panel) and MAP kinase-dependent (upper panel), with no role for PKA [32]. Activation of AP1 and C/EBP transcription factors by EPAC1 is sufficient to induce SOCS3 induction independently of MAP kinase signalling (lower panel) and therefore presents as a potential drug target

Based on these findings our hypothesis is that targeting of EPAC1 to the nuclear pore complex regulates c-Jun and C/EBP $\beta$ transcription factors, which control SOCS3 gene induction and suppression of proinflammatory cytokine signalling in VECs (Figure 1). Ongoing work is set to test this hypothesis and extend to identify the full range of human genes that are also regulated by this new, protective signalling pathway in VECs. These novel genetic foci will be associated with the protective actions of cyclic AMP and will therefore inform future strategies targeted at combating endothelial inflammation associated with CVD.

\section{Acknowledgement}

This work is funded by a project grant from the British Heart Foundation (Grant number PG/15/15/31316), awarded to TMP and SJY. 


\section{References}

1. Feng L, Matsumoto C, Schwartz A, Schmidt AM, Stern DM, et al. (2005) Chronic vascular inflammation in patients with type 2 diabetes: endothelial biopsy and RT-PCR analysis. Diabetes Care 28: 379-384.

2. Fanelli C, Aronoff R (1990) Restenosis following coronary angioplasty. Am Heart J 119: 357-368.

3. Ridker PM, Rifai N, Stampfer MJ, Hennekens CH (2000) Plasma concentration of interleukin-6 and the risk of future myocardial infarction among apparently healthy men. Circulation 101: 1767-1772.

4. Suzuki T, Ishiwata S, Hasegawa K, Yamamoto K, Yamazaki T (2000) Raised interleukin 6 concentrations as a predictor of postangioplasty restenosis. Heart 83: 578.

5. Shoji M, Furuyama F, Yokota Y, Omori Y, Sato T, et al. (2014) IL -6 mobilizes bone marrow-derived cells to the vascular wall, resulting in neointima formation via inflammatory effects. J Atheroscler Thromb 21: 304-312.

6. Naya M, Tsukamoto T, Morita K, Katoh C, Furumoto T, et al. (2007) Plasma interleukin -6 and tumor necrosis factor-alpha can predict coronary endothelial dysfunction in hypertensive patients. Hypertension research 30: 541-548.

7. Zhang W, Wang W, Yu H, Zhang Y, Dai Y, et al. (2012) Interleukin 6 underlies angiotensin II-induced hypertension and chronic renal damage. Hypertension 59: 136-144.

8. Sukovich DA, Kauser K, Shirley FD, DelVecchio V, Halks-Miller M, et al. (1998) Expression of interleukin-6 in atherosclerotic lesions of male ApoE-knockout mice: inhibition by 17beta-estradiol. Arterioscler Thromb Vasc Biol 18: 1498-1505.

9. Brod SA (2000) Unregulated inflammation shortens human functional longevity. Inflamm Res 49: 561-570.

10. Wei LH, Kuo ML, Chen CA, Chou CH, Lai KB, et al. (2003) Interleukin-6 promotes cervical tumor growth by VEGF-dependent angiogenesis via a STAT3 pathway. Oncogene 22: 1517-1527.

11. Jougasaki M, Ichiki T, Takenoshita Y, Setoguchi M (2010) Statins suppress interleukin-6-induced monocyte chemo-attractant protein-1 by inhibiting Janus kinase/signal transducers and activators of transcription pathways in human vascular endothelial cells. Br J Pharmacol 159: 1294-1303.

12. Heinrich PC, Behrmann I, Haan S, Hermanns HM, Müller-Newen G, et al. (2003) Principles of interleukin (IL)-6-type cytokine signalling and its regulation. Biochem J 374: 1-20.

13. Kleemann R, Zadelaar S, Kooistra $\mathrm{T}$ (2008) Cytokines and atherosclerosis: a comprehensive review of studies in mice. Cardiovasc Res 79: 360-376.

14. Sarwar N, Butterworth AS, Freitag DF, Gregson J, Willeit P, et al. (2012) Interleukin- 6 receptor pathways in coronary heart disease: a collaborative meta-analysis of 82 studies. Lancet 379: 1205-1213

15. Hingorani AD, Casas JP (2012) The interleukin-6 receptor as a target for prevention of coronary heart disease: a mendelian randomisation analysis. Lancet 379: 1214-1224.

16. Scheller J, Rose-John S (2012) The interleukin 6 pathway and atherosclerosis. Lancet 380: 338

17. Hou T, Tieu BC, Ray S, Recinos Iii A, Cui R, et al. (2008) Roles of IL-6 gp130 Signaling in Vascular Inflammation. Curr Cardiol Rev 4: 179-192.

18. Schuett H, Oestreich R, Waetzig GH, Annema W, Luchtefeld M, et al. (2012) Transsignaling of interleukin-6 crucially contributes to atherosclerosis in mice. Arterioscler Thromb Vasc Biol 32: 281-290.

19. Hashizume M, Hayakawa N, Suzuki M, Mihara M (2009) IL-6/sIL-6R trans-signalling, but not TNF-alpha induced angiogenesis in a HUVEC and synovial cell co-culture system. Rheumatol Int 29: 1449-1454.

20. Yoshimura A, Naka T, Kubo M (2007) SOCS proteins, cytokine signalling and immune regulation. Nat Rev Immunol 7: 454-465.

21. Tan JC, Rabkin R (2005) Suppressors of cytokine signaling in health and disease. Pediatr Nephrol 20: 567-575.
22. Sasaki A, Yasukawa H, Suzuki A, Kamizono S, Syoda T, et al. (1999) Cytokine-inducible $\mathrm{SH} 2$ protein-3 (CIS3/SOCS3) inhibits Janus tyrosine kinase by binding through the $\mathrm{N}$-terminal kinase inhibitory region as well as SH2 domain. Genes Cells 4: 339-351.

23. Williams JJ, Palmer TM (2012) Unbiased identification of substrates for the Epac1 - inducible E3 ubiquitin ligase component SOCS-3. Biochem Soc Trans 40: 215-218.

24. Liang X, He M, Chen T, Liu Y, Tian YL, et al. (2013) Multiple roles of SOCS proteins: differential expression of SOCS1 and SOCS3 in atherosclerosis. Int J Mol Med 31: 1066-1074.

25. Ortiz-Munoz G, Martin-Ventura JL, Hernandez-Vargas P, Mallavia B, Lopez-Parra V, et al. (2009) Suppressors of cytokine signaling modulate JAK/STAT -mediated cell responses during atherosclerosis. Arteriosclerosis, thrombosis, and vascular biology 29: 525-531.

26. Croker BA, Kiu H, Pellegrini M, Toe J, Preston S, et al. (2012) IL-6 promotes acute and chronic inflammatory disease in the absence of SOCS3. Immunol Cell Biol 90: 124-129.

27. Stahl A, Joyal JS, Chen J, Sapieha P, Juan AM, et al. (2012) SOCS3 is an endogenous inhibitor of pathologic angiogenesis. Blood 120: 2925-2929.

28. Jo D, Liu D, Yao S, Collins RD, Hawiger J (2005) Intracellular protein therapy with SOCS3 inhibits inflammation and apoptosis. Nat Med 11: 892-898.

29. Recio C, Oguiza A, Lazaro I, Mallavia B, Egido J, et al. (2014) Suppressor of cytokine signaling 1-derived peptide inhibits Janus kinase/signal transducers and activators of transcription pathway and improves inflammation and atherosclerosis in diabetic mice. Arterioscler Thromb Vasc Biol 34: 1953-1960.

30. Xiang S, Liu J, Dong N, Shi J, Xiao Y, et al. (2014) Suppressor of cytokine signaling 3 is a negative regulator for neointimal hyperplasia of vein graft stenosis. J Vasc Res 51: 132-143.

31. Sands WA, Woolson HD, Milne GR, Rutherford C, Palmer TM (2006) Exchange protein activated by cyclic AMP (Epac)-mediated induction of suppressor of cytokine signaling 3 (SOCS-3) in vascular endothelial cells. Mol Cell Biol 26: 6333-6346.

32. Yarwood SJ, Borland G, Sands WA, Palmer TM (2008) Identification of CCAAT/enhancer-binding proteins as exchange protein activated by cAMP-activated transcription factors that mediate the induction of the SOCS-3 gene. J Biol Chem 283: 6843-6853

33. Parnell E, Smith BO, Palmer TM, Terrin A, Zaccolo M, et al. (2012) Regulation of the inflammatory response of vascular endothelial cells by EPAC1. Br J Pharmacol 166: 434-446.

34. Borland G, Smith BO, Yarwood SJ (2009) EPAC proteins transduce diverse cellular actions of cAMP. Br J Pharmacol 158: 70-86

35. Netherton SJ, Sutton JA, Wilson LS, Carter RL, Maurice DH (2007) Both protein kinase A and exchange protein activated by cAMP coordinate adhesion of human vascular endothelial cells. Circ Res 101: 768-776.

36. Cullere X, Shaw SK, Andersson L, Hirahashi J, Luscinskas FW, (2005) Regulation of vascular endothelial barrier function by Epac, a cAMP activated exchange factor for Rap GTPase. Blood 105:1950-1955.

37. Fukuhara S, Sakurai A, Sano H, Yamagishi A, Somekawa S, et al. (2005) Cyclic AMP potentiates vascular endothelial cadherin - mediated cell-cell contact to enhance endothelial barrier function through an Epac-Rap1 signaling pathway. Mol Cell Bio 25: 136-146.

38. Kooistra MR, Corada M, Dejana E, Bos JL (2005) Epac1 regulates integrity of endothelial cell junctions through VE-cadherin. FEBS Lett 579: 4966-4972.

39. Birukova AA, Zagranichnaya T, Fu P, Alekseeva E, Chen W, et al. (2007) Prostaglandins PGE(2) and PGI(2) promote endothelial barrier enhancement via PKA- and Epac1/Rap1-dependent Rac activation. Exp Cell Res 313: 2504-2520.

40. Baumer Y, Drenckhahn D, Waschke J (2008) cAMP induced Rac 1mediated cytoskeletal reorganization in microvascular endothelium. Histochem Cell Biol 129: 765-778. 
Citation: Palmer TM, Yarwood SJ (2015) Regulation of Anti-Inflammatory Gene Expression in Vascular Endothelial Cells by EPAC1. J Cell Signal 1: 103. doi:10.4172/jcs.1000103

Page 4 of 4

41. Sehrawat S, Cullere X, Patel S, Italiano J Jr, Mayadas TN (2008) Role of Epac1, an Exchange Factor for Rap GTPases, in Endothelial Microtubule Dynamics and Barrier Function. Mol Biol Cell 19: 1261-1270.

42. Schmidt M, Sand C, Jakobs KH, Michel MC, Weernink PA (2007) Epac and the cardiovascular system. Curr Opin Pharmacol 7: 193-200.

43. Wiejak J, Dunlop J, Gao S, Borland G, Yarwood SJ (2012) Extracellular signal-regulated kinase mitogen-activated protein kinase-dependent SOCS-3 gene induction requires c-Jun, signal transducer and activator of transcription 3, and specificity protein 3 transcription factors. Mol Pharmacol 81: 657-668.

44. Wiejak J, Dunlop J, Yarwood SJ (2014) The role of c-Jun in controlling the EPAC1-dependent induction of the SOCS3 gene in HUVECs. FEBS Lett 588: 1556-1561
45. Gloerich M, Vliem MJ, Prummel E, Meijer LA, Rensen MG, et al. (2011) The nucleoporin RanBP2 tethers the cAMP effector Epac1 and inhibits its catalytic activity. J Cell Biol 193: 1009-1020.

46. Liu C, Takahashi M, Li Y, Dillon TJ, Kaech S, et al. (2010) The interaction of Epacl and Ran promotes Rapl activation at the nuclear envelope. Mol Cell Biol 30: 3956-3969.

47. Parnell E, Smith BO, Yarwood SJ (2015) The cAMP sensors, EPAC1 and EPAC2, display distinct subcellular distributions despite sharing a common nuclear pore localisation signal. Cell Signal 27: 989-996. 